\section{Evaluación de la microdeformación según diferentes espesores de la tabla ósea vestibular}

\section{Evaluation of micro strain in different thicknesses of the buccal bone plate}

\begin{abstract}
Resumen
La restauración implantosoportada en la zona maxilar anterior debe contar con un mínimo de tabla ósea vestibular que permita una transmisión de esfuerzos sin sobrecarga patológica. Objetivo: Evaluar la distribución de esfuerzos von Mises y microdeformaciones en diferentes espesores de la tabla vestibular en el segmento anterosuperior del maxilar. Métodos: Se utilizó el método de elementos finitos para modelar la zona anterior del maxilar superior con tres diferentes espesores de tabla ósea vestibular $(0,5,1$ y 1,5 mm), más una restauración implantosoportada, a la cual se aplicó una carga estática de $200 \mathrm{~N}$ (Newtons). Se evaluaron esfuerzos y microdeformaciones de las tablas óseas vestibulares a 2, 4 y $6 \mathrm{~mm}$ de la cresta ósea en dirección apical, tanto en el hueso esponjoso como en el cortical. Resultados: De acuerdo al método de elementos finitos se encontró que en el espesor $0,5 \mathrm{~mm}$ de tabla ósea vestibular (cortical) y a $2 \mathrm{~mm}$ de altura, se presentaron esfuerzos von Mises (31 $056 \mathrm{mpa}$ ) y microdeformación $(2154 \mu \varepsilon)$, y a medida que el espesor óseo aumentó a 1,5 mm en la altura de $2 \mathrm{~mm}$, se encontró (22 $913 \mathrm{mpa}$ y 1 $570 \mu \varepsilon$ ), esta misma tendencia se observó en las otras dos alturas. Conclusiones: Los mayores valores von Mises y de microdeformación se encontraron en la cresta ósea de la tabla vestibular de $0,5 \mathrm{~mm}$ de espesor y disminuyeron a medida que el volumen de hueso esponjoso aumentó en dirección apical.
\end{abstract}

Palabras clave: Análisis de elementos finitos; Hueso cortical; Implantes dentales.

\begin{abstract}
For an implant-supported restoration of the maxillary anterior area; the buccal bone plate should have a minimum thickness to ensure an optimal and predictable behavior of soft tissue. Objective: To evaluate the mechanical behavior of stress and microstrain (von Mises yield criterion); in different thicknesses of buccal maxillary bone plates. Methods: The finite element method was used for modeling the maxillary anterior area with three different buccal bone thickness $0.5,1$ and $1.5 \mathrm{~mm}$. An implant-supported restoration was modeled as well, to which a static load of $200 \mathrm{~N}$ was applied. Stress and microstrains were obtained in the buccal bone plates at 2, 4 and $6 \mathrm{~mm}$ from the alveolar crest towards the apical direction, both in cancellous and cortical bone. Results: Based on the finite element method, it was found that in the thickness of $0.5 \mathrm{~mm}$ of buccal bone plate (cortical) with $2 \mathrm{mms}$ height, results showed stress von Mises (31 $056 \mathrm{mpa}$ ) and displayed
\end{abstract}

\section{Artículo Original}

\author{
Julio Cesar Escobar Restrepo 1,a, Juan David \\ Serna Ceballos 1,a, Federico Latorre Correa ${ }^{1, a}$, \\ Junes Abdul Villarraga Ossa $1,2, \mathrm{~b}$ \\ 1 Universidad de Antioquia. Medellín, Colombia. \\ 2 Universidad Nacional de Colombia de Medellín. \\ Medellín, Colombia. \\ a Especialista en Odontología integral del adulto. \\ b Ingeniero Mecánico.
}

\section{Correspondencia:}

Julio Cesar Escobar Restrepo

Correo electrónico: cesar.escobar@udea.edu.co Universidad de Antioquia, Facultad de Odontología. Calle 67 No. 53-108. Medellín, Colombia.

\section{Coautores: \\ Juan David Serna Ceballos \\ juanserna2@msn.com \\ Federico La Torre Correa \\ latorre.federico29@gmail.com \\ Junes Abdul Villarraga Ossa \\ junes@udea.edu.co}

Conflicto de intereses: Los autores declaran no tener conflictos de interés.

Fuente de financiamiento: Autofinanciado

Fecha de recepción: 07/06/18

Fecha de aceptación: 14/07/18

( L Los autores. Este artículo es publicado por la revista Odontología Sanmarquina de la Facultad de Odontología, Universidad Nacional Mayor de San Marcos. Este es un artículo de acceso abierto, distribuido bajo los términos de la licencia Creative Commons Atribucion - No Comercia_Compartir Igual 4.0 Internacional. (http://creativecommons.org/licenses/by-nc-sa/4.0/) que permite el uso no comercial, distribución y reproducción en cualquier medio, siempre que la obra original sea debidamente citada. 
microstrains $(2154 \mu \varepsilon)$. As the thickness increased to $1.5 \mathrm{~mm}$ with the $2 \mathrm{~mm}$ height results stated (22913 mpa and $1570 \mu \varepsilon$ ). This same trend was observed in the other two heights. Conclusions: The highest values von Mises and micro deformation were found on the crest of the buccal plate with $0.5 \mathrm{~mm}$ thickness and decreasing as the volume of cancellous bone increased towards apical direction.

Keywords: Cortical bone; Dental implants; Finite element analysis.

\section{Introducción}

Factores como la enfermedad periodontal, caries dental, patologías bucomaxilofaciales y el traumatismo pueden ocasionar la pérdida dentaria ${ }^{1}$, lo que produce reabsorción de las tablas óseas, la cual es más marcada en la tabla vestibular que en la palatina debido a condiciones previas de espesor ${ }^{2,3}$. Con la pérdida de un incisivo central superior, la tabla ósea vestibular presenta una reabsorción en promedio del $30 \%$ a los 30 días, ocasionando deformidades anatómicas que comprometen la restauración del diente ${ }^{4}$. Una alternativa de rehabilitación oral, es la recuperación del diente mediante una protésis fija individual sobre un implante oseointegrado, que debe estar rodeado de un mínimo espesor óseo para lograr un resultado funcional y estético predecible 5,6 .

El esfuerzo producido en el hueso alrededor del implante puede generar microdeformación en la interfase ósea, debido a una diferencia de las propiedades mecánicas del hueso comparado con el implante, dicha deformación se mide con una variable física llamada "microstrain $\mu \varepsilon$ ” o microdeformación unitaria, definida como la relación entre la deformación y la longitud inicial bajo la acción de una carga. Frost ${ }^{7}$ catalogó las siguientes cinco formas de repuesta ósea frente a diferentes valores de microdeformación:

Desuso agudo: con valores de 0-50 $\mu \varepsilon$, Adaptación: con un intervalo de 50 a $200 \mu \varepsilon$ produciendo una disminución de la densidad ósea. Sobrecarga ligera: con intervalos de 200 a $1000 \mu \varepsilon$ donde la remodelación ósea está en equilibrio. Sobrecarga patológica: de 1000 a 3000 $\mu \varepsilon$ se estimula la actividad de remodelación. Fractura espontánea: entre 3000 a $10000 \mu \varepsilon$, generando fracturas por fatiga y reabsorción ${ }^{7}$.

Con estos criterios se pueden analizar las microdeformaciones óseas después de aplicar una carga, considerando el margen de seguridad de $2000 \mu \varepsilon^{8}$. Se ha estimado que el espesor de la tabla ósea en un mínimo de 1,5 a 2 $\mathrm{mm}$ favorece un mejor comportamiento frente al esfuerzo, permitiendo una estabilidad del tejido blando y una mejor estética gingival ${ }^{9}$. Estos espesores mayores de 1 $\mathrm{mm}$ han sido reportados en cortes tomográficos solo en el 9,8\% de los casos; El 80,1\% corresponde a un espesor de la tabla ósea menor de $1 \mathrm{~mm}$, siendo el espesor de 0,5 $\mathrm{mm}$ el que predomina a nivel crestal ${ }^{10}$.

La importancia de la estética en la rehabilitación de los dientes, la falta de investigaciones que consideren todos los tópicos alrededor de la misma, la necesidad de entender el comportamiento mecánico del hueso alrededor de los implantes dentales generan la necesidad de investigaciones que muestren este comportamiento y permitan construir protocolos de atención predecibles en el tiempo.

El propósito de esta investigación fue evaluar la distribución de los esfuerzos de von Mises y microdeformaciones en diferentes espesores de tabla vestibular en un central antero superior, mediante el método de elementos finitos.

\section{Métodos}

Se realizó un estudio experimental por simulación utilizando el método computarizado de elementos fini$\operatorname{tos}^{11,12}$. Para el modelo geométrico tridimensional se utilizó el software de diseño asistido por computador CAD (SolidWorks ${ }^{\bullet}$, U.S.A.). Una vez obtenido el modelado de los componentes, se exportaron al software de elementos finitos ANSYS 16 para el análisis del comportamiento mecánico de la estructura.

Se elaboraron modelos tridimensionales de los tres diferentes espesores óseos: $0,5 \mathrm{~mm}, 1 \mathrm{~mm}$ y $1,5 \mathrm{~mm}$, que incluyeron una cortical constante de $0,5 \mathrm{~mm}$ y el resto del espesor fue completado por hueso esponjoso, que determinó el espesor final. Estos modelos se realizaron con una calidad ósea tipo D2 caracterizada por presentar una cortical porosa y trabéculas finas, que corresponde al tipo de hueso más frecuentemente encontrado en la zona anterior del maxilar ${ }^{8}$. En este estudio numérico se asumió una oseointegración del $100 \%$, lo cual implicó el emplear contatos tipo "bonded".

Además se modeló un implante de diámetro $3,7 \mathrm{~mm}$ y $13 \mathrm{~mm}$ de longitud, pilar, tornillo, cofia cerámica, corona cerámica, y precarga con las siguientes características cada uno:

- Implante Zimmer ${ }^{\circledR}$ para diente anterior incisivo central superior: $13 \mathrm{~mm}$ de longitud, $3,7 \mathrm{~mm}$ de diámetro, 3,5 $\mathrm{mm}$ de diámetro de plataforma, hexágono interno 2,5 mm. Tapered Screw-Vent Implants TSVB13. Fabricante: Zimmer Dental1900 Aston Avenue Carlsbad, CA 92008-7308 USA. Referencia número: TSVB13. Casa Fabricante: Zimmer ${ }^{\circledR}$ Dental1900 Aston Avenue Carlsbad, CA 92008-7308 USA. Longitud cervical 2,5 mm, tercio medio $7,5 \mathrm{~mm}$, apical $3 \mathrm{~mm}$, distancia del paso 
de rosca $1,8 \mathrm{~mm}$, profundidad de la rosca 0,375 $\mathrm{mm}$, tipo de rosca triple en forma $\mathrm{V}$.

- Pilar de Zirconia Zimmer ${ }^{\circledR}$ con las siguientes características: Precontorneado, recto, conexión interna hexagonal, diámetro de $3,5 \mathrm{~mm}$, perfil de emergencia de $4,5 \mathrm{~mm}$, altura del margen vestibular 1 $\mathrm{mm}$, anillo de titanio en la interfaz con el implante. Referencia numero: ZRA341S. Casa Fabricante: Zimmer Dental1900 Aston Avenue Carlsbad, CA 92008-7308 USA.

- Cofia cerámica en disilicato de litio, con recubrimiento cerámico de E-max-Ceram.

- Corona completamente cerámica en disilicato de litio con un recubrimiento de capa de E-max-Ceram, cementada al pilar con cemento resinoso, para simular la estructura completa en condiciones clínicas normales.
Para obtener el esfuerzo y la microdeformación, se utilizaron las constantes elásticas: módulo de Young y coeficiente de Poisson, para cada uno de los elementos analizados (Tabla 1).

Se realizó un análisis elástico lineal estático donde se utilizó elementos tetraédricos de 10 nodos, con el fin de obtener una mejor aproximación de las geometrías de las partes, de esta manera se obtuvo una malla tridimensional para cada uno de los nueve cuerpos: hueso cortical, esponjoso, implante, anillo, pilar, tornillo, cofia cerámica, cemento y corona, que ensamblados forman 686597 nodos y 416581 elementos (Figuras 1 y 2).

La carga aplicada fue de $200 \mathrm{~N}$ en la superficie palatina a una angulación de $45^{\circ}$, que correspondió a la carga oclusal promedio reportada por la literatura para el sector anterior ${ }^{23}$ (Figura 3).

Tabla 1. Propiedades mecánicas de las estructuras y materiales modelados (MPa)

\begin{tabular}{llllcc}
\hline \multicolumn{1}{c}{ Material } & \multicolumn{2}{c}{ Módulo de Young } & & Coeficiente de Poisson \\
Fluencia
\end{tabular}
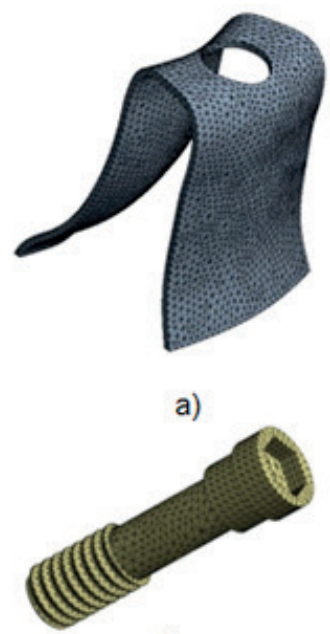

f)

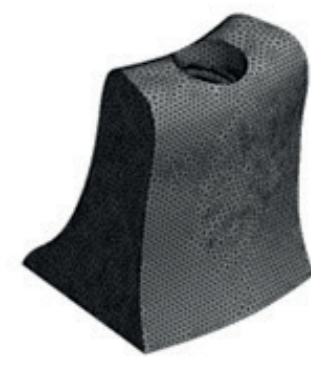

b)

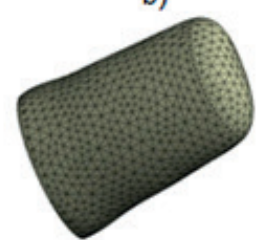

g)

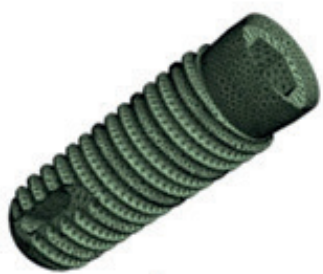

c)

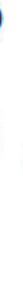

h)

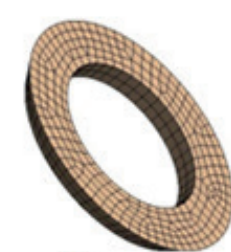

d)
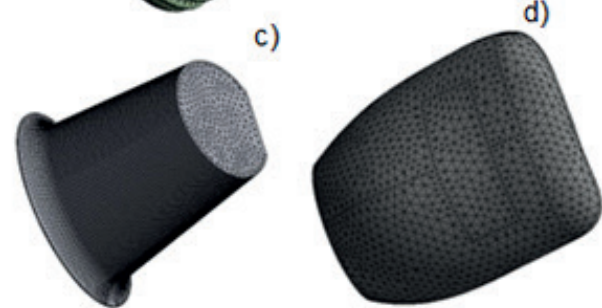

i)

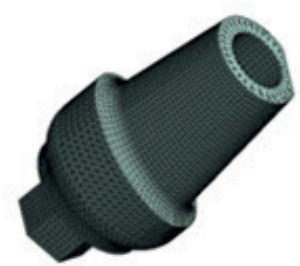

e)

Figura 1. Mallas de todos los elementos modelados: a) Hueso cortical, b) Hueso esponjoso, c) Implante, d) Aro, e) Pilar, f) Tornillo, g) Cemento, h) Estructura e i) Corona 


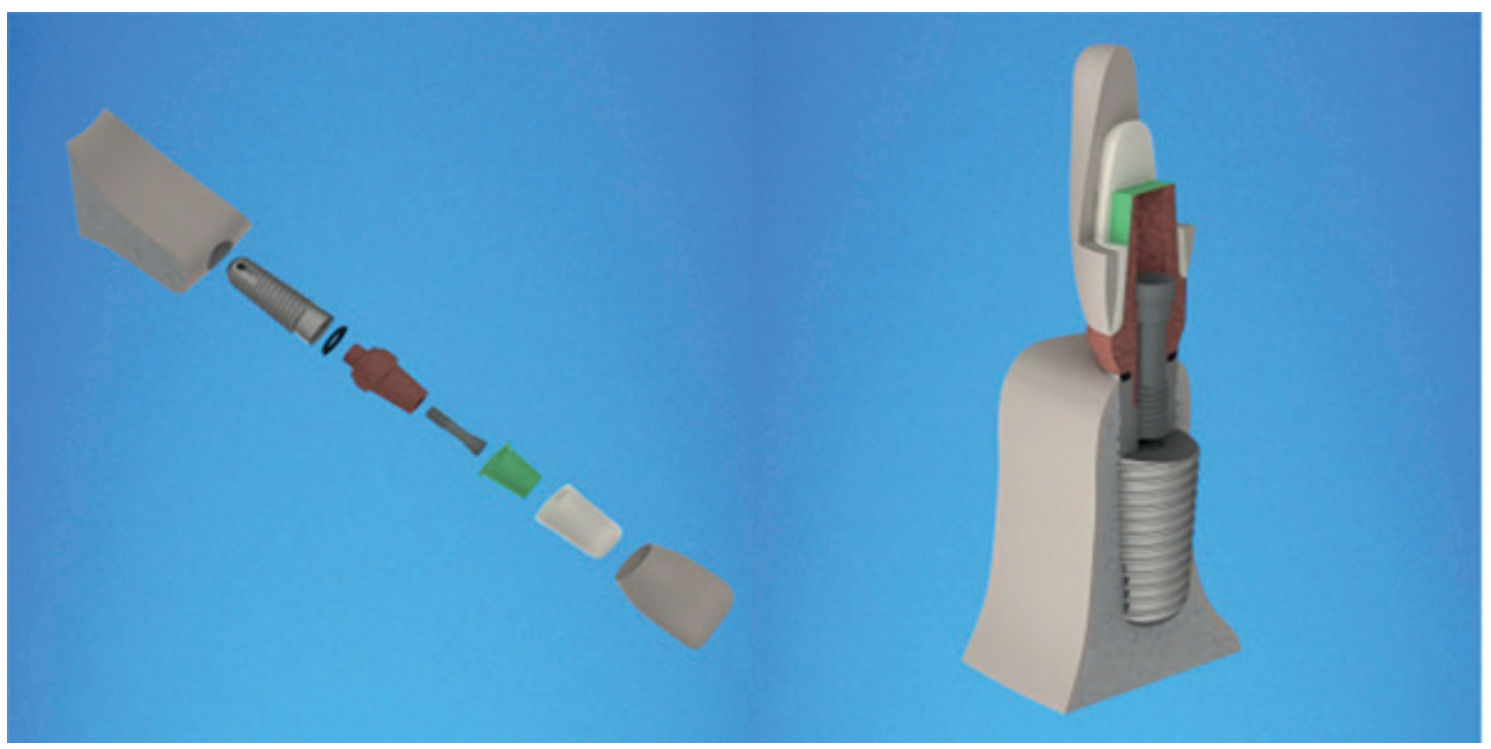

Figura 2. Visualización del sistema explosionado y del conjunto ensamblado

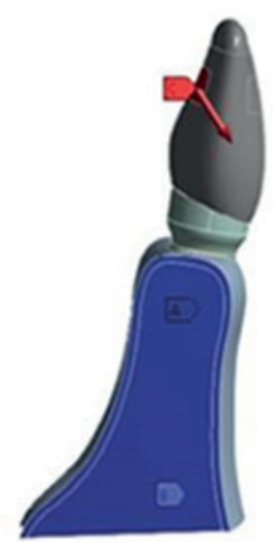

3A. $0,5 \mathrm{~mm}$

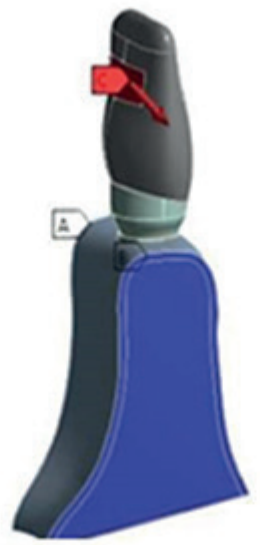

3B. $1 \mathrm{~mm}$

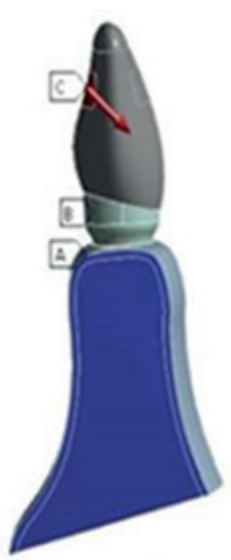

3C. $1,5 \mathrm{~mm}$

Figura 3. Dirección de fuerzas en cada espesor: 3A. 0,5 mm, 3B. 1 mm, 3C. 1,5 mm

Los esfuerzos y microdeformaciones se evaluaron en las tablas vestibulares de los tres modelos, tanto en los huesos esponjosos como corticales. Se tomó como referencia la cresta ósea y desde ahí se evaluaron a 2, 4 y $6 \mathrm{~mm}$ hacia apical, donde en cada una de estas zonas se tuvieron en cuenta cinco puntos, los cuales se promediaron para dar los resultados (Figura 4).

\section{Resultados}

\section{Distribución de esfuerzos von Mises en el hueso es- ponjoso y cortical}

La mayor distribución de esfuerzos se concentró en las crestas óseas en los tres modelos, con un mayor esfuerzo en el espesor de $0,5 \mathrm{~mm}$. Se observó un comportamiento lineal decreciente en la distribución de esfuerzos de los huesos esponjosos al aumentar el espesor y al desplazarse apicalmente, produciéndose mayor concentración de esfuerzos von Mises (2 $502 \mathrm{MPa}$ ) en las tablas vestibulares de $0,5 \mathrm{~mm}$ de espesor y en el área evaluada de $2 \mathrm{~mm}$. En el espesor de $1,5 \mathrm{~mm}$ se produjo menor concentración del esfuerzo (953 MPa) a una distancia de $6 \mathrm{~mm}$ apical a la cresta ósea (Tabla 2 y Figura 5).
Tabla 2. Esfuerzos von Mises (MPa) del hueso esponjoso

\begin{tabular}{|c|c|c|c|}
\hline \multirow{2}{*}{ Altura } & \multicolumn{3}{|c|}{ Espesor } \\
\hline & $0,5 \mathrm{~mm}$ & $1 \mathrm{~mm}$ & $1,5 \mathrm{~mm}$ \\
\hline $2 \mathrm{~mm}$ & 2502 & 2332 & 2234 \\
\hline $4 \mathrm{~mm}$ & 1621 & 1368 & 1253 \\
\hline $6 \mathrm{~mm}$ & 1298 & 993 & 953 \\
\hline
\end{tabular}

El hueso cortical presentó una distribución de esfuerzos von Mises igual que el esponjoso, pero en mayor magnitud, un comportamiento lineal decreciente en la distribución de esfuerzos, al aumentar el espesor y al desplazarse apicalmente, produciendo los menores esfuerzos von Mises (13740 MPa) en el espesor de 1,5 mm en la distancia más apical $6 \mathrm{~mm}$, y el mayor esfuerzo von Mises (31056 MPa) se produjo en el espesor de $0,5 \mathrm{~mm}$ en la distancia más crestal de 2 mm (Tabla 3 y Figura 6).

\section{Micro deformaciones en el hueso cortical y esponjoso}

La distribución de las microdeformaciónes fueron similares para los diferentes espesores, con una mayor concentración en la cresta ósea. El hueso esponjoso presentó 

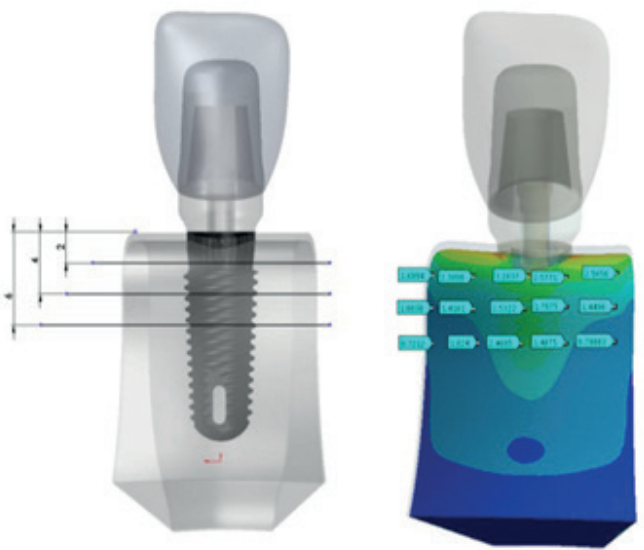

Figura 4. Ubicación de las zonas evaluadas en dirección corono-apical

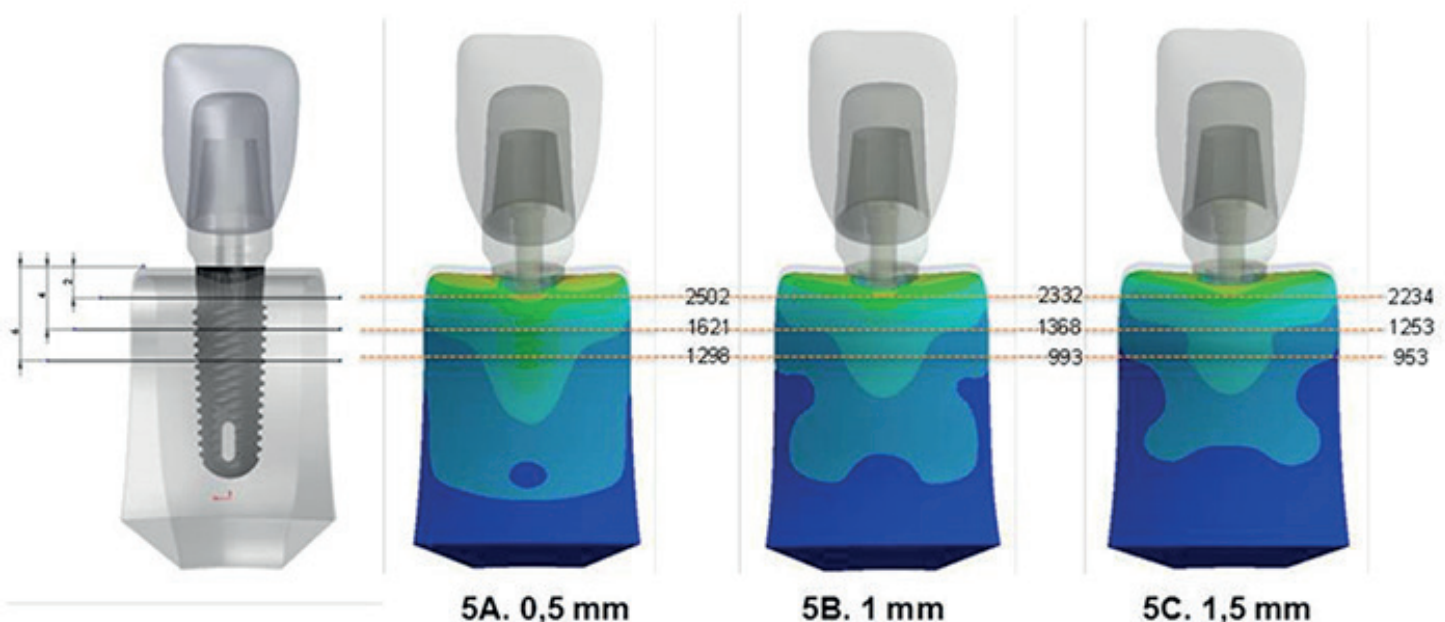

Figura 5. Esfuerzo de von Mises promedio en hueso esponjoso a las diferentes alturas de $2 \mathrm{~mm}, 4 \mathrm{~mm}$ y $6 \mathrm{~mm}$ en los espesores de tabla ósea vestibular: 5 A. $0,5 \mathrm{~mm}, 5$ B. $1 \mathrm{~mm}$ y 5 C. $1,5 \mathrm{~mm}$

Tabla 3. Esfuerzos von Mises (MPa) del hueso cortical

\begin{tabular}{rccc}
\hline \multirow{2}{*}{ Altura } & \multicolumn{3}{c}{ Espesor } \\
\cline { 2 - 4 } & $\mathbf{0 , 5} \mathbf{~ m m}$ & $\mathbf{1 ~} \mathbf{~ m m}$ & $\mathbf{1 , 5} \mathbf{~ m m}$ \\
\hline $2 \mathrm{~mm}$ & 31056 & 28759 & 22913 \\
$4 \mathrm{~mm}$ & 23571 & 22629 & 17908 \\
$6 \mathrm{~mm}$ & 16795 & 16115 & 13740 \\
\hline
\end{tabular}

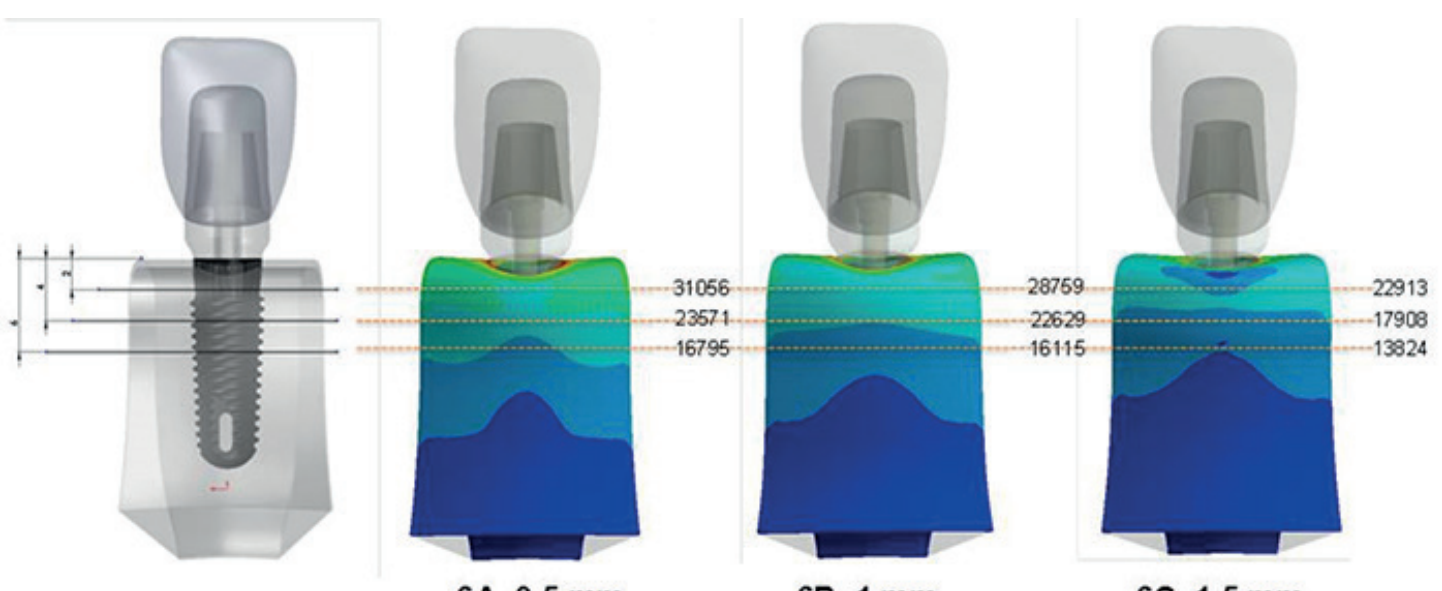

6A. $0,5 \mathrm{~mm}$

6B. $1 \mathrm{~mm}$

6C. $1,5 \mathrm{~mm}$

Figura 6. Esfuerzo de von Mises promedio en hueso cortical a las diferentes alturas de $2 \mathrm{~mm}, 4 \mathrm{~mm}$ y $6,0 \mathrm{~mm}$ en los espesores de tabla ósea vestibular: 6 A. $0,5 \mathrm{~mm}, 6 \mathrm{~B} .1 \mathrm{~mm}$ y $6 \mathrm{C} .1,5 \mathrm{~mm}$ 
un comportamiento lineal decreciente, donde el menor valor de micro deformación $(654 \mu \varepsilon)$ se encontró en el espesor de $1,5 \mathrm{~mm}$ en la ubicación más apical de $6 \mathrm{~mm}$, y el mayor valor de micro deformación $(1567 \mu \varepsilon)$ se encontró en el espesor de $0,5 \mathrm{~mm}$ en la ubicación más crestal de $2 \mathrm{~mm}$ (Tabla 4 y Figura 7).

En el hueso cortical la microdeformación tuvo un comportamiento lineal decreciente, dependiente del espesor y la ubicación de la zona evaluada, donde el menor valor

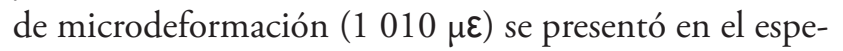
sor de $1,5 \mathrm{~mm}$ en la distancia más apical de $6 \mathrm{~mm}$, y el mayor valor de micro deformación $(2154 \mu \varepsilon)$ se presentó en el espesor de $0,5 \mathrm{~mm}$ en la distancia más crestal de $2 \mathrm{~mm}$ (Tabla 5 y Figura 8).

\section{Discusión}

El método de elementos finitos (MEF) ha demostrado ser una herramienta útil en la investigación de sistemas complejos que son difíciles de estandarizar durante investigaciones in vitro e in vivo ${ }^{24}$. En este estudio se utilizó este método, evaluando la distribución de esfuerzos y las microdeformaciónes para cada uno de los elementos de la restauración y considerando los diferentes espesores óseos, $0,5 \mathrm{~mm}, 1 \mathrm{~mm}$ y $1,5 \mathrm{~mm}$ de las tablas óseas vestibulares con un implante en la zona anterior del maxilar. De acuerdo a los resultados de estos modelos, los huesos esponjosos y corticales presentaron un comportamiento lineal decreciente a medida que aumentó el espesor, es decir, a mayor espesor óseo de la tabla vestibular, menor fue la concentración de esfuerzos y la microdeformación.

La mayor concentración de los esfuerzos Von Mises se ubicó a $2 \mathrm{~mm}$ de la cresta ósea, esto concuerda con lo encontrado en otros estudios ${ }^{25-27}$, donde la distribución de los esfuerzos se concentró en la cresta, especialmente en huesos de calidad tipo D2 y D3. Esto se entiende por la ausencia de ligamento periodontal y la ubicación

Tabla 4. Microdeformaciones $(\mu \varepsilon)$ del hueso esponjoso

\begin{tabular}{rccc}
\hline \multirow{2}{*}{ Altura } & \multicolumn{3}{c}{ Espesor } \\
\cline { 2 - 4 } & $\mathbf{0 , 5} \mathbf{~ m m}$ & $\mathbf{1 ~} \mathbf{~ m m}$ & $\mathbf{1 , 5} \mathbf{~ m m}$ \\
\hline $2 \mathrm{~mm}$ & 1567 & 1474 & 1372 \\
$4 \mathrm{~mm}$ & 1092 & 964 & 841 \\
$6 \mathrm{~mm}$ & 921 & 767 & 654 \\
\hline
\end{tabular}

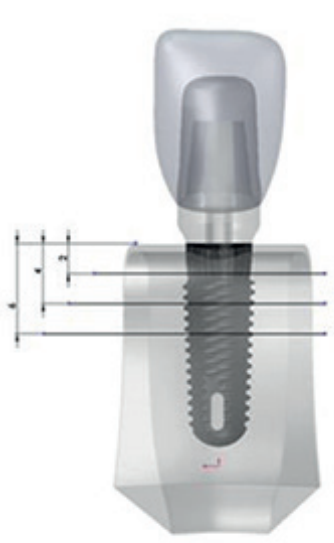

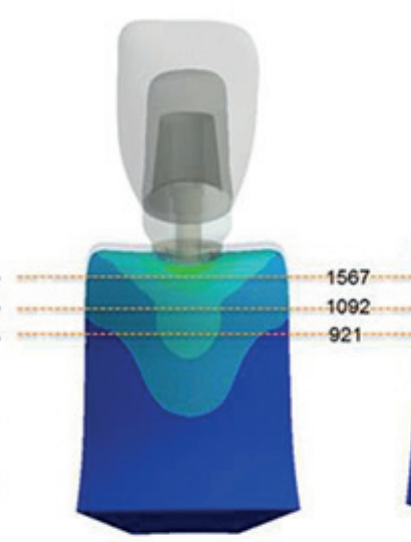

7A. $0,5 \mathrm{~mm}$

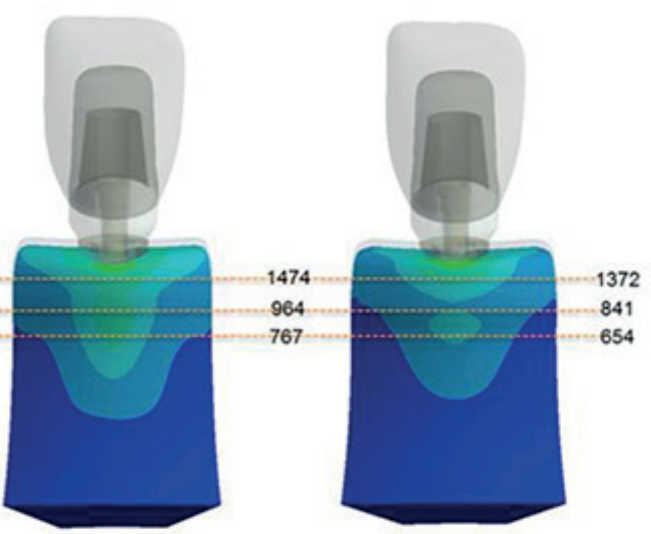

7B. $1 \mathrm{~mm}$
7C. $1,5 \mathrm{~mm}$

Figura 7. Micro deformaciones en el hueso esponjoso a diferentes alturas en los diferentes espesores de tabla vestibular: 7A. 0,5 $\mathrm{mm}, 7 \mathrm{~B} .1 \mathrm{~mm}$ y 7 C. $1,5 \mathrm{~mm}$

Tabla 5. Microdeformaciones $(\mu \varepsilon)$ del hueso cortical

\begin{tabular}{lccc}
\hline \multirow{2}{*}{ Altura } & \multicolumn{3}{c}{ Espesor } \\
\cline { 2 - 4 } & $\mathbf{0 , 5} \mathbf{~ m m}$ & $\mathbf{1 ~} \mathbf{~ m m}$ & $\mathbf{1 , 5} \mathbf{~ m m}$ \\
\hline $2 \mathrm{~mm}$ & 2154 & 2039 & 1570 \\
$4 \mathrm{~mm}$ & 1723 & 1658 & 1343 \\
$6 \mathrm{~mm}$ & 1291 & 1191 & 1010 \\
\hline
\end{tabular}




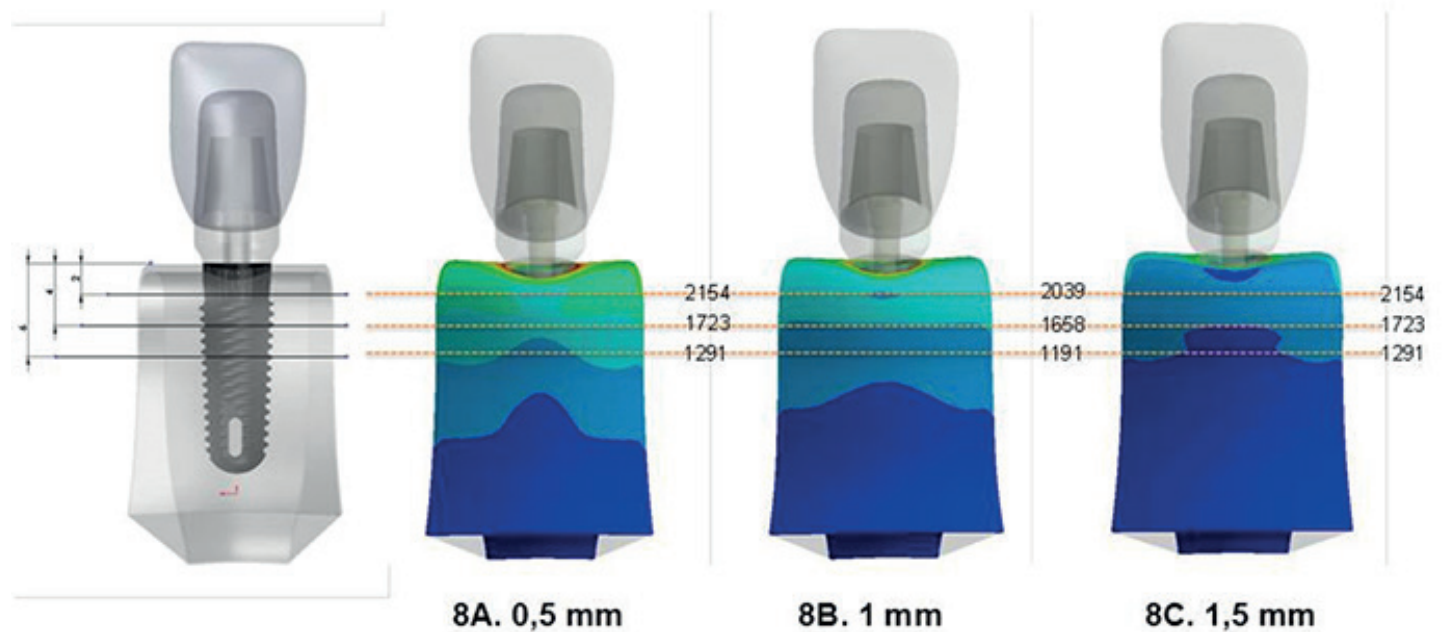

Figura 8. Micro deformaciones en el hueso cortical a diferentes alturas en los diferentes espesores de tabla vestibular: 8A. 0,5mm, $8 B .1 \mathrm{~mm}$ y 8 C. $1,5 \mathrm{~mm}$

más coronal del centro de rotación en el implante. Los mayores esfuerzos de $2502 \mathrm{MPa}$ en huesos esponjosos y $31056 \mathrm{MPa}$ para huesos corticales se observaron en el espesor de $0,5 \mathrm{~mm}$. Esta diferencia considerable entre el hueso esponjoso y cortical, se debe a las propiedades mecánicas de cada tipo de hueso y a las características estructurales como la densidad ósea y la porosidad.

Este estudio consideró un espesor de hueso cortical constante de $0,5 \mathrm{~mm}$ para los diferentes espesores, con mayores volúmenes de hueso esponjoso en los espesores de 1 y $1,5 \mathrm{~mm}$. Los valores más altos de microdeformación se encontraron en el hueso cortical a $2 \mathrm{~mm}$ de la cresta ósea. Se apreció una disminución significativa en la microdeformación del hueso esponjoso al pasar de 0,5 o $1 \mathrm{~mm}$ a $1,5 \mathrm{~mm}$ de espesor, sugiriendo un mejor comportamiento en la medida que el cortical se acompañe con un mayor volumen de hueso esponjoso.

A diferencia del hueso cortical, en el hueso esponjoso no se apreciaron diferencias significativas en los espesores de $0,5 \mathrm{~mm}, 1 \mathrm{~mm}$ y $1,5 \mathrm{~mm}$ a $2 \mathrm{~mm}$ de la cresta ósea, con valores de $1567 \mu \varepsilon, 1474 \mu \varepsilon$ y $1372 \mu \varepsilon$ respectivamente, manteniéndose en el rango del hueso cortical a un espesor de $1,5 \mathrm{~mm}$.

Los resultados mostraron la importancia del volumen de hueso esponjoso en la adaptación mecánica a la deformación antes de presentarse fractura espontánea y mantenerse en las zonas de adaptación $(50 \mu \varepsilon$ a 1500 $\mu \varepsilon$,) y zona de sobre carga ligera $(1500 \mu \varepsilon$ a $3000 \mu \varepsilon)$, asumidos como fisiológicos en la literatura ${ }^{7,8,28}$.

Ramos et al ${ }^{29}$, realizaron un modelo diferente al de esta investigación, ya que no se especificó los valores de hueso cortical y esponjoso, y se simuló un hueso de calidad D3, a pesar de esto encontraron resultados similares en la distribución de los esfuerzos von Mises y micro deformación en el hueso cortical. En los modelos con una mayor proporción corona-implante, mostraron valores de microdeformación de 1020 a $1520 \mu \varepsilon$ al someter- se a una carga oblicua de $100 \mathrm{~N}$, concentrados a nivel crestal. Estos resultados coinciden con esta investigación por estar en valores de carga ligera y estando cerca al valor de $1570 \mu \varepsilon$ del hueso cortical en el espesor de $1,5 \mathrm{~mm}$ de este estudio. Esto se puede explicar por el papel mecánico del hueso esponjoso en la distribución de esfuerzos en ambos estudios, un modelado óseo en el estudio de Ramos et al ${ }^{29}$, de un hueso tipo D3 y una mayor cantidad de hueso esponjoso en el espesor de 1,5 $\mathrm{mm}$ de este estudio.

Chou et $a l^{30}$, encontraron mayor microdeformación en el hueso a nivel de la cresta ósea en el modelo de implante de diámetro amplio con un espesor óseo menor. $\mathrm{Al}$ igual que Ramos et al ${ }^{30}$ y este estudio, los valores de microdeformación estuvieron en la zona de adaptación $(1000 \mu \varepsilon-3000 \mu \varepsilon)$.

Tanto para este estudio, como para el de Ramos et al. ${ }^{29} \mathrm{y}$ Chou et al. ${ }^{30}$, no se obtuvieron valores de microdeformación mayores a $3000 \mu \varepsilon$, que de acuerdo a las diferentes formas de respuesta ósea propuesta por Frost, corresponde a la zona de sobrecarga patológica, donde se generan micro fracturas y pérdida ósea.

Según los resultados de este estudio, el volumen de hueso esponjoso dentro los diferentes espesores evaluados, influye en los valores de microdeformación, siendo menor en el espesor de $1,5 \mathrm{~mm}$, donde existe un mayor volumen de hueso esponjoso, que permite una mayor posibilidad de distribuir el esfuerzo en una mayor cantidad de área y de volumen.

Con las limitantes de este estudio, los resultados permitieron concluir que el espesor de $1,5 \mathrm{~mm}$ brindó un margen de seguridad en la distribución del esfuerzo y valor de microdeformación en un rango fisiológico. Para los espesores de 0,5 y $1 \mathrm{~mm}$, los valores de microdeformación estuvieron cercanos a los $3000 \mu \varepsilon$, indicativo de un aumento de actividad en la remodelación, lo que aumenta la densidad ósea (sobrecarga patológica); sin 
embargo, ninguno de los espesores presento valores de microdeformaciones que indicaran posible fractura por fatiga y reabsorción.

Estos resultados deben ser confirmados con estudios clínicos e in vitro, ya que este es un estudio matemático de simulación de un evento biológico, que permite una información basado en unas propiedades mecánicas de los tejidos y elementos incorporados pero no es un evento real.

\section{Referencias bibliográficas}

1. Petersen PE. The World Oral Health Report 2003: continuous improvement of oral health in the 21st century - the approach of the WHO Global Oral Health Programme. Community Dent Oral Epidemiol. 2003;31(1):3-23.

2. Araújo M, Lindhe J. Dimensional ridge alterations following tooth extraction. An experimental study in the dog. J Clin Periodontol. 2005;32(2):212-8.

3. Araújo M, Linder E, Wennström J, Lindhe J. The influence of Bio-Oss Collagen on healing of an extraction socket: an experimental study in the dog. Int J Periodontics Restorative Dent. 2008;28(2):123-35.

4. Nevins M, Stein J. The Placement of Maxillary Anterior Implants. En: Nevins, Myron and Mellonig, James T, Editores. Implant Therapy: Clinical Approaches and Evidence of Success, Volume II. Illinois: Quintessence Publishing; 1998. p. $111-127$.

5. Raigrodski AJ, Chiche GJ. The safety and efficacy of anterior ceramic fixed partial dentures: A review of the literature.J Prosthet Dent. 2001;86(5):520-5.

6. Wang HL, Kiyonobu K, Neiva RF. Socket augmentation: rationale and technique. Implant Dent. 2004;13(4):286-96.

7. Frost HM. Bone "mass" and the "mechanostat": a proposal. Anat Rec. 1987 ;219(1):1-9.

8. Misch CE. Bone density: A key determinant for treatment planning. Part I: diagnosis and rationale. En: Misch CE,Editores. Contemporary Implant Dentistry. $3^{\text {a }}$ ed. Ed Mosby. Canada: Elsevier; 2009.

9. Elian N, Ehrlich B, Jalbout ZN, Classi AJ, Cho SC, Kamer AR, et al. Advanced concepts in implant dentistry: creating the "aesthetic site foundation". Dent Clin North Am. 2007 ;51(2):547-63.

10. Braut V, Bornstein MM, Belser U, Buser D. Thickness of the anterior maxillary facial bone wall- a retrospective radiographic study using cone beam computed tomography. Int J Periodontics Restorative Dent. 2011;31(2):125-31.

11. DeTolla DH, Andreana S, Patra A, Buhite R, Comella B. Role of the finite element model in dental implants. J Oral Implantol. 2000;26(2):77-81.

12. Geng JP, Tan KB, Liu GR. Application of finite element analysis in implant dentistry: a review of the literature. J Prosthet Dent. 2001;85(6):585-98.

13. Peterson J, Wang Q, Dechow PC. Material properties of the dentate maxilla. Anat Rec A Discov Mol Cell Evol Biol. 2006;288(9):962-72.
14. O'Mahony AM, Williams JL, Spencer P. Anisotropic elasticity of cortical and cancellous bone in the posterior mandible increases peri-implant stress and strain under oblique loading. Clin Oral Implants Res. 2001;12(6):648-57.

15. Kayabaşı O, Yüzbasıoğlu E, Erzincanlı F. Static, dynamic and fatigue behaviors of dental implant using finite element method. Adv Eng Softw. 2006;37(10):649-58.

16. Niinomi M. Mechanical biocompatibilities of titanium alloys for biomedical applications. J Mech Behav Biomed Mater 2008;1:30-42.

17. Papanagiotou HP, Morgano SM, Giordano RA, Pober R. In vitro evaluation of low-temperature aging effects and finishing procedures on the flexural strength and structural stability of Y-TZP dental ceramics. J Prosthet Dent. 2006;96(3):154-64

18. RelyXUnicem 2 Automix - Technical Data Sheet [Internet]. [citado 2013 may 19]. Recuperado a partir de http://multimedia. 3 m.com/mws/mediawebserver?mwsId=66666UF6EVsSyXTtnxf218TXEVtQEVs6EVs6EVs6E666666--\&fn=rx_u2_auto_tds.pdf

19. Luthardt RG, Holzhüter MS, Rudolph H, Herold V, Walter MH.CAD/CAM-machining effects on Y-TZP zirconia. Dent Mater. 2004;20(7):655-62.

20. IPS e.maxCeramScientificDocumentation [Internet]. [citado 2013 may 19]. Recuperado a partir de: http:// www.infinident.com/ecomaXL/get_blob.php?name=IPS_e.max_Ceram_Scientific_Doc.pdf

21. Ozkurt Z, Kazazoglu E, Unal A. In vitro evaluation of shear bond strength of veneering ceramics to zirconia. Dent Mater J. 2010;29(2):138-46.

22. IPS e.max Press. Scientific Documentation [Internet]. [citado 2013 may 19]. Recuperado a partir de: http:// www.ivoclarvivadent.com/zoolu-website/media/document/9808/IPS+e-max+Press

23. Morneburg TR, Proschel PA. In vivo forces on implants influenced by occlusal scheme and food consistency. Int J Prosthodont. 2003;16(5):481-6.

24. Romeed SA, Fok SL, Wilson NH. A comparison of 2D and 3D finite element analysis of a restored tooth. J Oral Rehabil. 2006;33(3):209-15.

25. Schrotenboer J, Tsao YP, Kinariwala V, Wang HL. Effect of microthreads and platform switching on crestal bone stress levels: a finite element analysis. J Periodontol. 2008;79(11):2166-72

26. Chu CM, Huang HL, Hsu JT, Fuh LJ. Influences of internal tapered abutment designs on bone stresses around a dental implant: three-dimensional finite element method with statistical evaluation. J Periodontol. 2012;83(1):111-8.

27. Borie E, Orsi IA, Noritomi PY, Kemmoku DT. Three-Dimensional Finite Element Analysis of the Biomechanical Behaviors of Implants with Different Connections, Lengths, and Diameters Placed in the Maxillary Anterior Region. Int J Oral Maxillofac Implants. 2016;31(1):101-10.

28. Baggi L, Cappelloni I, Di Girolamo M, Maceri F, Vairo G. The influence of implant diameter and length on stress distribution of osseointegrated implants related to 
crestal bone geometry: a three-dimensional finite element analysis. J Prosthet Dent. 2008;100(6):422-31.

29. Ramos F, Santiago JF, de Faria DA, de Oliveira GB, de Souza VE, Marques H, et al. Biomechanical influence of crown-to-implant ratio on stress distribution over internal hexagon short implant:3-D finite element analysis with statistical test. J Biomech. 2015;48(1):138-45
30. Chou HY, Müftü S, Bozkaya D. Combined effects of implant insertion depth and alveolar bone quality on periimplant bone strain induced by a wide-diameter, short implant and a narrow-diameter, long implant. J Prosthet Dent. 2010;104(5):293-300. 
